\title{
From the world to word order: the link between conceptual structure and language
}

\author{
Jennifer Culbertson (jennifer.culbertson@ed.ac.uk) \\ Marieke Schouwstra (Marieke.Schouwstra@ed.ac.uk) \\ Simon Kirby (simon.kirby@ed.ac.uk) \\ Centre for Language Evolution, University of Edinburgh \\ 3 Charles St, Edinburgh EH8 9AD, UK
}

\begin{abstract}
The world's languages exhibit striking diversity. At the same time, recurring linguistic patterns suggest the possibility that this diversity is shaped by features of human cognition. One well-studied example is word order in complex noun phrases (like 'these two red vases'). While many orders of these elements are possible, a subset appear to be preferred. It has been argued that this order reflects a single underlying representation of noun phrase structure, from which preferred orders are straightforwardly derived (e.g., Cinque, 2005). Building on previous experimental evidence using artificial language learning, we show that these preferred orders arise not only in existing languages, but also in improvised sequences of gestures produced by English speakers. We then use corpus data from a wide range of languages to argue that the hypothesized underlying structure of the noun phrase might be learnable from statistical features relating objects and their properties conceptually. Using an information-theoretic measure of strength of association, we find that adjectival properties (e.g. "red") are on average more closely related to the objects they modify (e.g. "wine"), than numerosities (e.g. "two"), which are in turn more closely related to the objects they modify than demonstratives (e.g., "this"). It is exactly those orders which transparently reflect this - by placing adjectives closest to the noun, and demonstratives farthest away - which are more common across languages, and preferred in our silent gesture experiments. These results suggest that our experience with objects in the world, combined with a preference for transparent mappings from conceptual structure to linear order, can explain constraints on noun phrase order.
\end{abstract}

\section{Introduction}

One of the oldest debates in linguistics concerns whether the languages of the world share a set of core invariant properties reflecting universal features of human cognition. At the center of this debate is a tension between the diversity we see when we look across languages, and the similarities which crop up when they are analyzed under a certain lens. This tension, between linguistic diversity on the one hand, and universal organizing principles on the other, is on full display in one of the simplest linguistic structures we use: the noun phrase. Given just a noun (e.g., "vases") and three common categories of words which modify it - a demonstrative (e.g., "these"), a numeral (e.g., "two"), and an adjective (e.g., "blue") — there are already 24 possible ways of ordering the words to make a phrase, almost all of which are found in some language. For example, the English order is "these two blue vases"; in Thai, it would be the equivalent of "vases blue two these"; in Vietnamese, it would be "these two vases blue"; in Basque, it would be "two vases blue those"; and so on. Yet there remains a small subset of orders which no language appears to use systematically. 

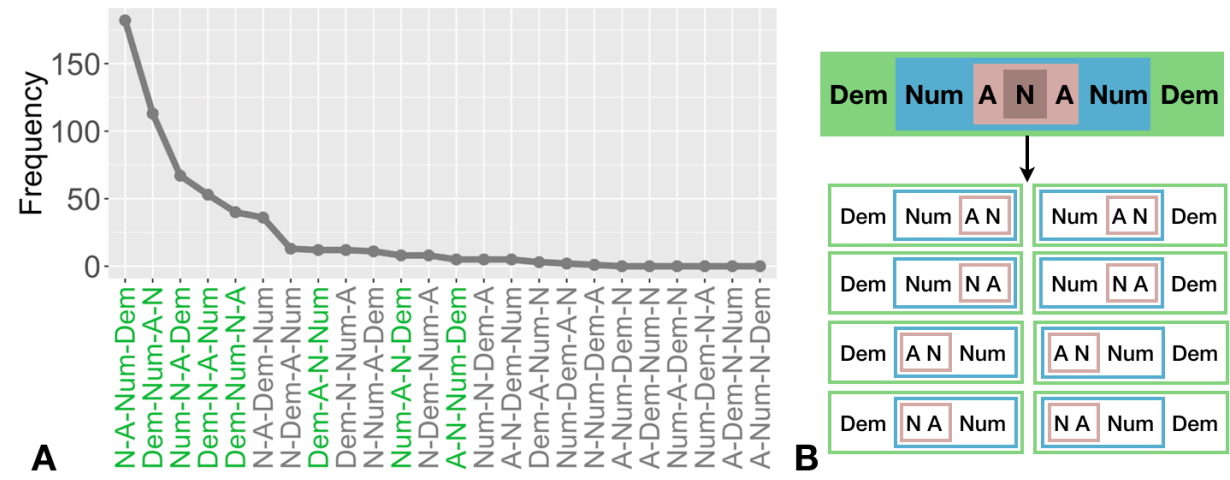

Figure 1: A Estimated frequency of each of the 24 possible orders (based on counts of languages sampled in Dryer, 2018, N=noun, A=adjective, Num=numeral, Dem=demonstrative), with homomorphic patterns highlighted in green, showing a clear preference for homomorphism across languages. B Schematic representation of sub-units or constituents in the noun phrase and the resulting eight homoomorphic orders, which preserve this underlying structure.

For example, we currently know of no language which systematically uses the equivalent of "blue two these vases" or "blue these vases two". 1

Linguists have argued that these missing patterns offer evidence of a universal organizing principle underlying how noun phrases are built (Cinque, 2005; Abels \& Neeleman, 2012; Steddy \& Samek-Lodovici, 2011; Dryer, 2018; Steedman, 2018). As careful analyses of noun phrase order exist for only a small sample of the world's languages (around 700 in Dryer, 2018), any one pattern could be absent by chance (Piantadosi \& Gibson, 2014). Here, we focus not on which patterns are currently attested in the world's languages, but instead on the frequency differences among the 24 possible orders. The dramatically skewed distribution is shown in fig. 1A.

\section{Homomorphism and noun phrase word order}

What sort of organizing principle might explain why some noun phrase orders are so much more common than others? All current accounts start from the idea that adjectives, numerals, and demonstratives are not created equal. Rather, they differ in how they combine with each other. To illustrate this, take a complex word like "speakers", comprised of a lexical root speak and two morphemes -er and $-s$. The meaning of the word reflects how these two morphemes combine with the root; speak combines with -er first, creating a noun, "speaker". This larger unit is then pluralized by combining with $-s$. The order of semantic composition is here preserved in the linear order - the morpheme that combines its meaning with the noun root first is closer to the root. This same idea can be applied to see how elements in the noun phrase (here multiple words) combine to form a coherent meaning. The adjective forms a unit with the noun first (i.e., "vase" is modified by the property "blue"). The resulting unit then combines with the numeral (i.e. the numerosity of the blue vases is specified), and finally that unit combines with the demonstrative (e.g., the group of blue vases is located in space relative to the speaker). This composition order is typically assumed to be reflected in the syntax (Adger, 2003; Alexiadou, Haegeman, \& Stavrou, 2007), creating an underlying hierarchical structure in which each sub-unit forms a syntactic constituent. ${ }^{2}$ Just as

\footnotetext{
${ }^{1}$ Different typological samples and sampling techniques reported in the literature result in slightly different frequency estimations, including some discrepancies as to which orders remained unattested (Cinque, 2005; Cysouw, 2010; Dryer, 2018). Importantly, the shape of the distribution and remains the same.

${ }^{2}$ Basic constituency tests also show that in a phrase like "these blue vases", "blue vases" is a constituent (it can be replaced by "ones"). This order of composition is supported by formal semantic accounts of different modifier
} 
in the case of morpheme order, the linear order of words in a noun phrase can in principle reflect this underlying structure, or not. An order which does will have the adjective placed closer to the noun, and the demonstrative farthest away. Following A. Martin, Abels, Adger, and Culbertson (submitted), we refer to these as homomorphic orders ${ }^{3}$. There are eight such orders, shown in fig. 1B, and they make up the bulk of the most frequently attested orders in fig. 1A.

The notion of homomorphism-a transparent mapping between underlying structure (i.e., the compositional units described above) and linear order-thus describes a kind of hidden similarity between languages which on the surface appear to be different. This is exactly the kind of innate, universal organizing principle posited by many linguists, but it is worth unpacking what this might mean. If the explanandum is the frequency differences among noun phrase orders, then two potential organizing principles must be involved. First, a universal preference for transparent mappings between underlying structure and linear order. A universal preference is not a hard-and-fast constraint, but rather one that is, by hypothesis, present in all humans but violable in their languages (e.g., as in Culbertson, Smolensky, \& Wilson, 2013)). After all, the majority of languages are homomorphic, but non-homomorphic languages can arise and are learnable. In addition, there is reason to believe that transparent mappings are preferred across cognition, reflecting a domain-general preference for simplicity in learning (Culbertson \& Kirby, 2016; Chater \& Vitányi, 2003).

The second piece of the puzzle is the underlying structure itself, in particular, the compositional units described above. Some linguists have argued that constraints on noun phrase order provide evidence for a specialized innate linguistic system (Cinque, 2005; Abels \& Neeleman, 2012; Steedman, 2018). More explicitly, the claim is that the categories Adjective, Numeral, Demonstrative, and Noun are innately known (or expected) by language learners, who tacitly know how they combine semantically, and build an underlying syntactic structure based on this (Adger, 2003). From the moment children map words in their language onto these categories they will expect Adjectives to combine with the Noun before Numerals, and Demonstratives to combine last.

Here we explore these two hypothesized universals - a general cognitive preference for homomorphism, and a specialized underlying structure for the noun phrase (reflecting semantic composition and/or syntactic constituency). First, we show that when English speakers improvise a system of gestural communication, their gesture orders are systematically homomorphic. This supplements existing experimental evidence for a homomorphism bias in humans (Culbertson \& Adger, 2014; A. Martin et al., submitted), and supports the claim that this bias is at play in explaining noun

types. For example, most adjectives are treated as predicates that combine with the noun (e.g., B. H. Partee, 1987), numerals are widely analyzed functions from nominal predicates to countable units (e.g., B. Partee, 1988; Kratzer \& Heim, 1998), and demonstratives as functions mapping nominal predicates to individuals (e.g., (Elbourne, 2008)). This also aligns with functionally-oriented work on the noun phrase, which argues that nouns and adjectives appear closer together syntactically than numerals, because they are closer semantically (Hurford, 1987; J. Rijkhoff, 1990; Jan Rijkhoff, 2004). All previous accounts of noun phrase word order cited here therefore assume this underlying structure (either in terms of syntax or semantics, or both).

${ }^{3}$ The term isomorphic is used in Culbertson and Adger (2014), but as A. Martin et al. (submitted) point out it is more accurate to call these orders homomorphic, reserving isomorphic for the two most frequent orders- $\mathrm{N}-\mathrm{Adj}-\mathrm{Num}-$ Dem and Dem-Num-Adj-N. The latter are the only two orders from which it is possible to fully recover the underlying structure. In Dem-Num-N-Adj, by contrast, the surface order does not contradict the structure illustrated by 1B, but it is not possible to recover the relations between e.g., Dem and Adj. Therefore such orders are homomorphic but not isomorphic. The especially high frequency of isomorphic orders could then be explained by an independent preference for word order harmony. Indeed, both Culbertson, Smolensky, and Legendre (2012), and Dryer (2018) argue that there is a preference for consistent placement of modifiers before or after the noun. The two orders N-Adj-Num-Dem and Dem-Num-Adj-N are the most common because they are homomorphic and have consistent order of modifiers relative to the noun. Of the non-homomorphic orders that are attested, many are harmonic (e.g., N-A-Dem-Num, N-Dem-A-Num, N-Dem-Num-A). 
phrase order in established languages. Then, we use an information-theoretic measure of strength of association to argue that the universal structure which shapes noun phrase order can be learned from observing the world, rather than reflecting (innate) linguistic knowledge. Specifically, we argue that statistical features relating objects and their properties can be used to build up a conceptual representation of the noun phrase. The skewed distribution of word orders across languages thus comes from a pressure to be homomorphic combined with a universal conceptual structure which mirrors properties of the world around us.

\section{Experiment 1}

While previous accounts of noun phrase word order have implicitly assumed that homomorphic orders are a kind of default (e.g., Abels \& Neeleman, 2012), recent work has sought to provide direct behavioral evidence for an homomorphism bias using laboratory experiments (Culbertson \& Adger, 2014; A. Martin, Abels, Ratitamkul, \& Culbertson, to appear; A. Martin et al., submitted). For example, Culbertson and Adger (2014) trained English speakers on a pseudo-artificial language: participants saw English phrases with a pre-nominal modifier (e.g., "blue vases", "two cows", "these shoes") and heard a translation into the new language, where modifiers were post-nominal (e.g., "vases blue", "darts two", "shoes these"). Crucially, the phrases they were trained on only ever had a single modifier (either an adjective or a numeral or a demonstrative); no evidence was given about the relative order of modifiers in the new language. At test, participants were shown these held-out, multiple-modifier phrases in English (e.g. "these two vases", "two blue vases", "these blue vases"), and asked to guess how they should be translated in the new language. One option was consistent with the surface order of modifiers in English, but not homomorphic (e.g., "vases these blue"). The other featured the reverse order of modifiers from English, but was homomorphic (e.g., "vases blue these"). Participants consistently chose the homomorphic option over the alternative, despite the latter being more probable given their experience with strings of modifiers in English. This is in line with an homomorphism bias operating on a universal underlying structure. However, the results could also reflect transfer at a more abstract level; they may have learned from English that surface order should be homomorphic, and may have transferred this to post-nominal modifiers in the experiment.

Stronger evidence would come from showing that speakers of a non-homomorphic language still show a bias favouring homomorphic order. However, this is challenging for two reasons. First, very few well-documented languages are in fact non-homomorphic. Second, widespread bilingualism means that even if we were to test those speakers, they are still very likely to have experience with an homomorphic language (either English or otherwise). Instead, we attempt to bypass the effects of prior linguistic knowledge to the degree possible, by using the silent gesture paradigm. In silent gesture experiments, participants with no knowledge of a sign language must improvise a way to convey information using only their hands and no speech. This method has been popular in exploring biases underlying basic word order, showing that when participants use gestures to describe simple events (e.g., "Alex kicked the ball"), they often bypass the dominant order of their native language (Goldin-Meadow, So, Özyürek, \& Mylander, 2008; Futrell et al., 2015), and take the semantic properties of the information to be conveyed into account (Gibson et al., 2013; Hall, Mayberry, \& Ferreira, 2013; Schouwstra \& de Swart, 2014; Schouwstra, Smith, \& Kirby, 2016). Here we use this method to investigate biases in noun phrase order. We expect that, as in experiments on basic word order, gestures will not simply recapitulate English order. Rather, if a bias for transparent mapping between underlying structure and linear order is at play even when participants are improvising in a modality distinct from their previous language experience, we expect their gesture order to be homomorphic. 


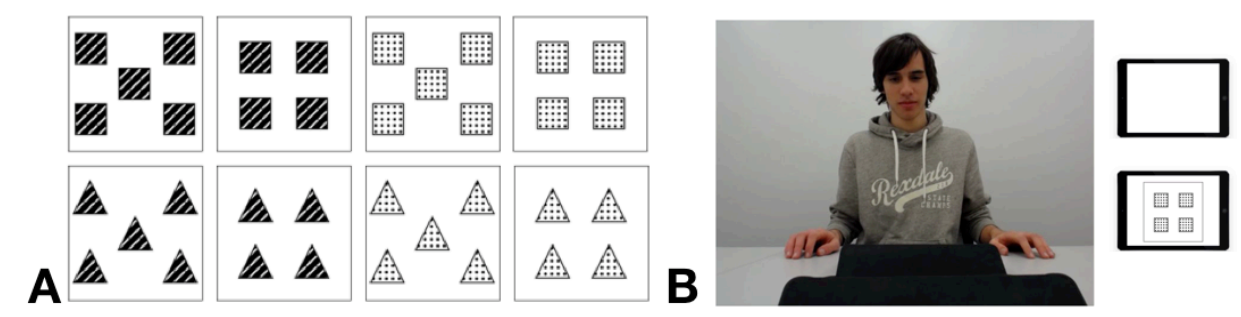

Figure 2: A Stimulus set for Experiment 1. B Set-up of the experiment, with participant seated in front of two iPads displaying images (e.g., as shown alongside).

\subsection{Method}

\subsubsection{Participants}

Participants were 20 native-English speakers. None had previous knowledge of any sign language. ${ }^{4}$

\subsubsection{Materials}

The stimulus set consisted of images of squares or triangles. They appeared in groups of four or five and were either striped or spotted fig. 2A. Location relative to the gesturer was represented by two iPads that displayed the images, one of which was directly in front of the participant, and the other about an arm's length away. The 8 different images, presented on two different iPads, together formed 16 total stimulus items, which were each presented twice, in random order for each participant.

\subsubsection{Procedure}

Participants were seated at a table across from the experimenter, with the two iPads in front of them (see fig. 2B). They were filmed using a Logitech camera connected to a Macbook Air which controlled stimulus presentation over a networked server. Before starting, participants were shown the set of 8 images they would have to gesture as printed pictures. They were told they should describe each image using their hands, without any speech, so that someone watching the recorded video would be able to work out what they were seeing. In both experiments, there was a brief break after the first block of 16 trials.

\subsubsection{Coding}

Example gesture clips are shown in fig. 3. Gestures were coded by identifying which part of the gesture corresponded to information associated with each conceptual piece of the image: the object corresponded to the $\mathrm{N}$ (oun), the texture/size to the $\mathrm{A}$ (djective), the numerosity to Num(eral), and the location to Dem(onstrative). In some cases, additional information other than that corresponding to the N, A, Num, or Dem was conveyed (e.g. about the spatial arrangement of the objects in an image). This was ignored for the purposes of coding order. If Dem, A, Num or $\mathrm{N}$ were omitted, or gestures included simultaneous information (i.e., if the object shape was gestured while simultaneously indicating numerosity) no order was coded for the relevant element(s). ${ }^{5}$ Gesture strings were then coded as homomorphic or not, based on whether they

\footnotetext{
${ }^{4}$ Experiments were approved by the PPLS Ethics Committee at the University of Edinburgh. All participants gave consent prior to beginning.

${ }^{5}$ In a small number of cases ( 7 total) simultaneous gestures meant we could not determine whether the order was homomorphic or not (e.g., in a gesture string with Dem followed by a simultaneous gesture incorporating N A and Num, the relative order of A and Num is not clear).
} 


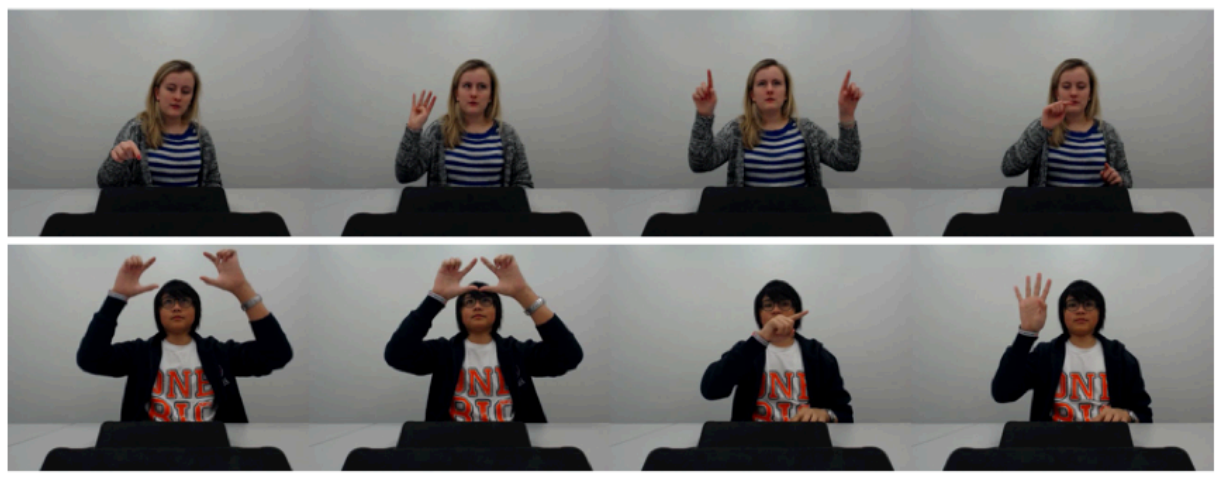

Figure 3: Clips from two participants illustrating experimental set-up with proximal and distal iPads and example gestures (Top: stimulus "four spotted squares" on proximal iPad, order Dem-Num-N-A; Bottom: stimulus "four striped triangles" on distal iPad, order Dem-N-A-Num).

were consistent with one of the eight orders shown in Figure 1B. For example, Dem-N-A-Num was homomorphic as was N-A-Num (with omitted Dem); Dem-N-Num-A by contrast was nonhomomorphic as was N-Num-A. Gestures were coded once by the first author, and a second time by an independent coder (blind to the hypothesized difference between conditions in Experiment 2 ). Agreement on gesture order was $82 \%$, on gesture homomorphism was $92 \%$. All instances of disagreement were resolved by discussion with a third coder.

\subsection{Results}

As predicted, gesture strings were overwhelmingly homomorphic, indeed only $2 \%$ were nonhomomorphic ( $11 \%$ were ambiguous, e.g., due to gesture combinations, or repetitions). The frequency of gesture orders and how often a given pattern was used in the majority of a participant's gestures (i.e., as the participant's default order) are shown in fig. $4 \mathrm{~A}$ and $\mathrm{B}$ respectively. All of the latter are homomorphic. The most commonly used patterns were Dem-Num-N-A and Dem-N-ANum, neither of which are the basic noun phrase word order used in English (Dem-Num-A-N). This raises the question, as in other studies employing the silent gesture paradigm, of what these gesture strings correspond to linguistically. While they could correspond to more complex noun phrases (e.g., akin to the English phrase "these two squares with spots"), they could also correspond more closely to sequences of sentences (e.g., "On that iPad, there are two squares. The squares have spots"). Alternatively, it could be that there is not a clear equivalent to speech, at least in the mind of the gesturer. While we will return to this point below, these data suggest that, regardless of the linguistic status of these gestures, the information conveyed is organized temporally in a way that is homomorphic to the hypothesized underlying structure.

\subsection{Learning conceptual structure from the world}

To summarize, typological evidence points to the high frequency of homomorphic orders relative to non-homomorphic alternatives. Artificial language learning experiments using spoken language suggest that English speakers assume homomorphic orders of post-nominal modifiers (Culbertson \& Adger, 2014; A. Martin et al., to appear; A. Martin et al., submitted). This same population innovates homomorphic orders when improvising gesture sequences. All three sources of evidence point to the existence of an homomorphism bias, assuming an underlying structure in which the adjective combines with the noun first, then the numeral, then the demonstrative. We now turn to the origins of the underlying structure itself, assumed to be innate in previous accounts (Cinque, 2005; Abels \& Neeleman, 2012; Steddy \& Samek-Lodovici, 2011; Steedman, 2018; Dryer, 2018). We 


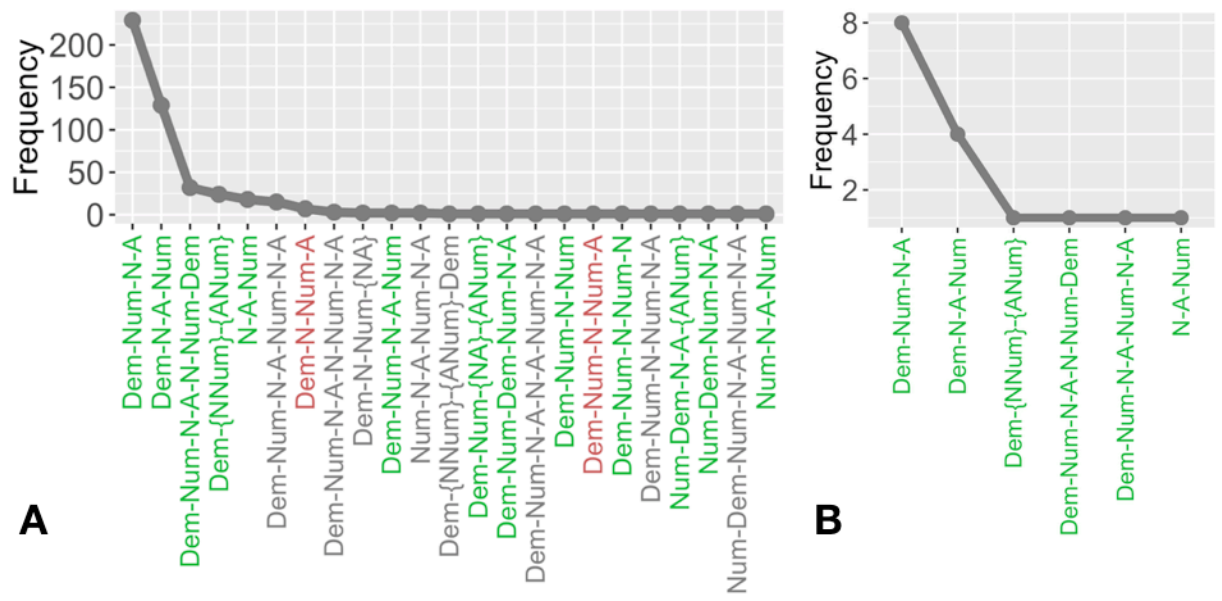

Figure 4: A Raw frequency of each order produced by participants. Green patterns are homomorphic; red patterns are non-homomorphic; gray patterns are ambiguous; parenthetical elements were gestured simultaneously. B Patterns that were used in the majority of at least one participant's gestures (x-axis), and the number of participants using it (y-axis). The overwhelming majority of orders produced were homomorphic, none straightforwardly reflect the basic English order (Dem-Num-A-N).

propose instead that it can be learned by observing the environment: objects in the world (expressed linguistically by the category Noun) are more closely related to their properties (expressed by Adjective) than their numerosities (expressed by Numeral), which are in turn more closely related to objects than their location and/or relation to the speaker (expressed by Demonstrative). Intuitively, differences in strength of association among modifier types can be seen by considering common objects in the world. For example, wine is closely associated with its color (e.g., red or white); skyscrapers are closely associated with their height; Dalmatians are closely associated with their texture (e.g., spotted). It is trivial to come up with other such examples of objects which are closely associated with particular properties. By contrast, examples of objects which are closely associated with their numerosity are difficult to come by. Some things typically come in pairs, like shoes; or dozens, like eggs, however most objects are not closely associated with a numerosity. Objects are even less likely to be associated with their location and/or relation to the speaker; in fact these concepts are by their very nature changeable.

Strength of association can be formalized in information-theoretic terms as pointwise mutual information (PMI), given in (1). PMI tells us whether a given pair of elements co-occur more than would be expected from their base frequency rates. ${ }^{6}$ If wine co-occurs with the property red more often than it would in a world in which objects and properties combined freely, then this pair will have high PMI. PMI for a pair of elements will be zero when the two elements are completely independent of one another, and negative when they co-occur less than would be expected by their base rates. Our claim is that on average, objects and their properties have higher PMI than objects and their numerosities, which in turn have higher PMI than objects and their location and/or relation to the speaker. If children track these differences in conceptual closeness while learning about the world, and use them to build up a conceptual structure representing how these elements relate to each other, then this structure should mirror that shown in fig. 1B. The domain-general bias for homomorphism will in turn drive preferences in the ordering of the linguistic categories

\footnotetext{
${ }^{6} \mathrm{PMI}$ is a symmetric measure: it therefore captures the intuition not only of a strong association between a Dalmatian and its spots, but also the possibility that spots are very likely to bring to mind Dalmatians.
} 
Noun, Adjective, Numeral, and Demonstrative.

$$
p m i=\log \frac{p(x, y)}{p(x) p(y)}
$$

To test our hypothesis, we estimated the PMI of (Noun, Modifier) pairs from dependency-parsed natural language corpora. We use corpora rather than, e.g., image sets, because they provide a representative sample of the kinds of concepts which frequently appear (and are salient) in our environment. By contrast, large image sets are almost exclusively tagged with object names, and do not include, for example, information about an object's relation to a speaker. Note however, that using corpora means there will be some influence of linguistic categories, which are imposed on the world by our minds (see Appendix A for additional discussion). We calculate the base frequency rates of nouns and modifiers, and then count how often individual adjectives, numerals, and demonstrative modify individual nouns. The critical comparison is the average PMI values across (Noun, Modifier) pairs, for each modifier type. To ensure our results are not influenced by any particular language, we replicate this using corpora from 24 languages across a number of families, plus all English corpora in CHILDES (Sagae, Davis, Lavie, MacWhinney, \& Wintner, 2007) (see Appendix A for details).

\subsection{Methods}

We first extracted all dependencies involving a noun and an adjective, a noun and a numeral, or a noun and a demonstrative. We excluded all singleton pairs to prevent PMI values from being skewed by low frequency items (Jurafsky \& Martin, to appear), and then estimated the probabilities of each pair, and each member of the pair. The probabilities were calculated using maximum likelihood estimation. The precise implementation of the pmi calculation was as in (2), where $n$ is a noun, $m$ is a modifier, and $t(n, m)$ is a noun and a modifier in a dependency of type $t$ (either A, Num, or Dem). In other words, the probability of a given modifier is based on the set of modifiers of that type modifying a head noun, and the probability of the noun is based on the set of nouns that have that type of modifier. ${ }^{7}$

$$
p m i=\log \frac{p(n, m \mid t(n, m))}{p(n \mid t(n, m)) p(m \mid t(n, m))}
$$

\subsection{Results}

Fig. 5 shows average PMI values for each modifier type across all language corpora. As predicted, on average, (Noun, Adjective) pairs have the highest PMI, (Noun, Demonstrative) pairs have the lowest PMI, and (Noun, Numeral) pairs fall in between. A linear mixed-effects regression model with language as a random effect confirms this (using Helmert contrast coding, with adjective as the default level: adjectives vs. numerals: $\beta=-2.44 \pm 0.02, p<0.001$; mean of adjectives and numerals vs. demonstratives: $\beta=-0.16 \pm 0.01, p<0.001)$. Based on this information-theoretic measure of strength of association, properties (conveyed by Adjectives) are on average more closely associated with objects (conveyed by Nouns) than numerosities (conveyed by Numerals), which are

\footnotetext{
${ }^{7}$ Note that this is not the only way to implement the PMI calculation. For example, it is also possible to calculate probabilities over the set of all the phrases with a modifier modifying a head noun (i.e., across the whole set of noun phrases modified by A, Num and Dem). The implementation reported here generally lowers PMI across the board relative to this alternative. However, both ways of computing PMI give the same results: adjectives pairs have the highest PMI, and demonstrative pairs the lowest. For additional discussion of the relationship between PMI and entropy, see SI.
} 

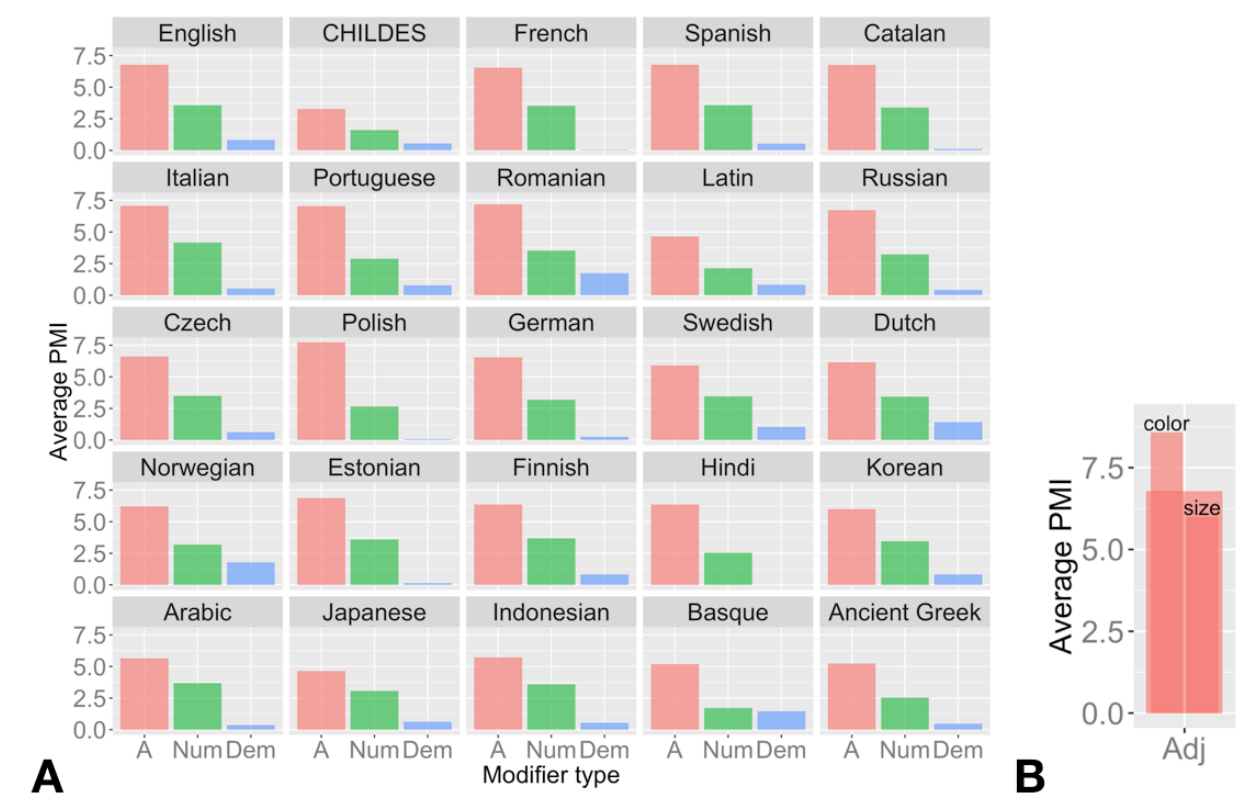

Figure 5: A Average PMI values across corpora of 25 languages confirming that, according to our measure, properties are more closely related to objects than numerosities are, and numerosities are more closely related to objects than location/discourse status is. B Distribution of PMI values for adjectives in the English corpora highlighting subset of color and texture vs. size adjectives; by this measure, color/texture properties are more closely related to objects on average than size properties.

in turn more closely related with object than location or status relative to the speaker (conveyed by Demonstratives). This information is available to children, who can use it along with many other sources of statistical information from the world around them to build up conceptual knowledge (as they do with linguistic knowledge, (Saffran, 2003)). Specifically, under the assumption that what is talked about in these corpora reflects what is in the world around us, average differences in the degree of relatedness between different modifier types may lead to a representation of conceptual structure as in fig. 1A. In principle, this same source of statistical knowledge could also help children to categorize modifier types; in addition to other semantic (and syntactic) differences, strength of association with the modified object is also a source of information which distinguishes adjectives from numerals from demonstratives.

\section{Experiment 2}

What we have suggested so far is that differences in conceptual closeness to the noun across modifier types lead to differences in underlying conceptual structure. This conceptual structure in turn is reflected in the distribution of linear orders found across languages (mediated by a bias for homomorphism). While this idea has not been applied to these distinct modifier types, something like it has been suggested as an explanation for patterns of adjective ordering (e.g., Champollion, 2006; Seiler, 1991; Bouchard, 2002), among other factors (see Scontras, Degen, \& Goodman, 2017). For example, J. E. Martin (1969) argued that when a phrase contains multiple adjectives, their relative order is influenced by "the degree to which they denote properties which are inherent or essential to the denoted object". Fig. 5B shows average PMI for color/texture adjectives (which 

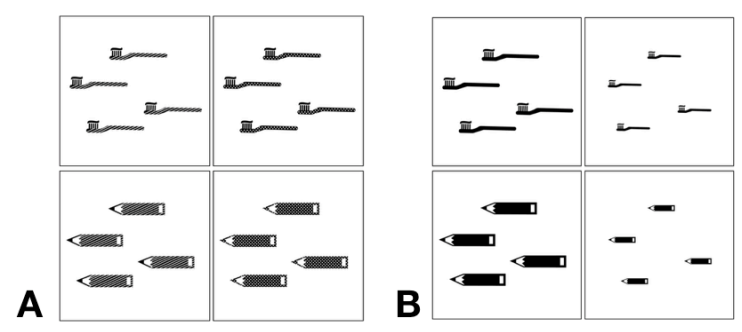

Figure 6: Stimulus set for Experiment 2.

tend to pattern the same) and size adjectives in the English corpora. ${ }^{8}$ This correctly predicts that color/texture adjectives should generally be ordered closer to the noun than size (e.g., "small green vase" is preferred to "green small vase" in English and other languages, (Cinque, 1993; Scott, 2002; Truswell, 2009)). If these more subtle differences in average PMI connect directly to homomorphism, our prediction is that adjectives which differ in their average PMI should differ in the extent to which they are ordered homomorphically in spontaneous gesture strings. To evaluate this, we conducted a second silent gesture experiment, directly comparing the improvised gesture strings produced by participants when object properties were higher PMI textures ("striped" and "spotted", as in Experiment 1), or lower PMI sizes ("small" and "large").

\subsection{Method}

\subsubsection{Participants}

Participants were 40 native-English speakers. Participants were randomly assigned to the texture or size condition, therefore they gestured only high or low PMI adjectives. None had previous knowledge of any sign language.

\subsubsection{Materials}

The objects featured in Experiment 2 were toothbrushes or pencils (see fig. 6. In Experiment 1 (where we used squares and triangles) we observed participants using combined gestures (e.g., shape and texture simultaneously). We anticipated that this would be even more common with size (e.g., conveying large and square as a combined gesture). In order to discourage participants from using simultaneous gestures in Experiment 2 we therefore used objects - pencil and toothbrushwhich are typically conveyed using associated actions rather than by depicting the shape Padden, Hwang, Lepic, and Seegers, 2015. As in Experiment 1, objects appeared in groups of four or five. In the high PMI condition, objects were either striped or spotted (see fig. 6A). We used texture rather than color because improvising a gesture for a color is relatively difficult compared to a texture like striped or spotted. In the low PMI (size) condition, objects were either big or small (see fig. 6B). Location relative to the gesturer was represented by two iPads that displayed the images, one of which was directly in front of the participant, and the other about an arm's length away (see fig. 2B. The 8 different images, presented on two different iPads, together formed 16 total stimulus items, which were presented twice, in random order for each participant.

\subsubsection{Procedure}

The procedure was identical to Experiment 1 with one exception: at the end of Experiment 2, we asked participants to reflect on the gesture order they used, and we gave them a single trial to describe using English.

\footnotetext{
${ }^{8}$ For additional information about PMI calculations for specific adjective classes, see Appendix A.
} 


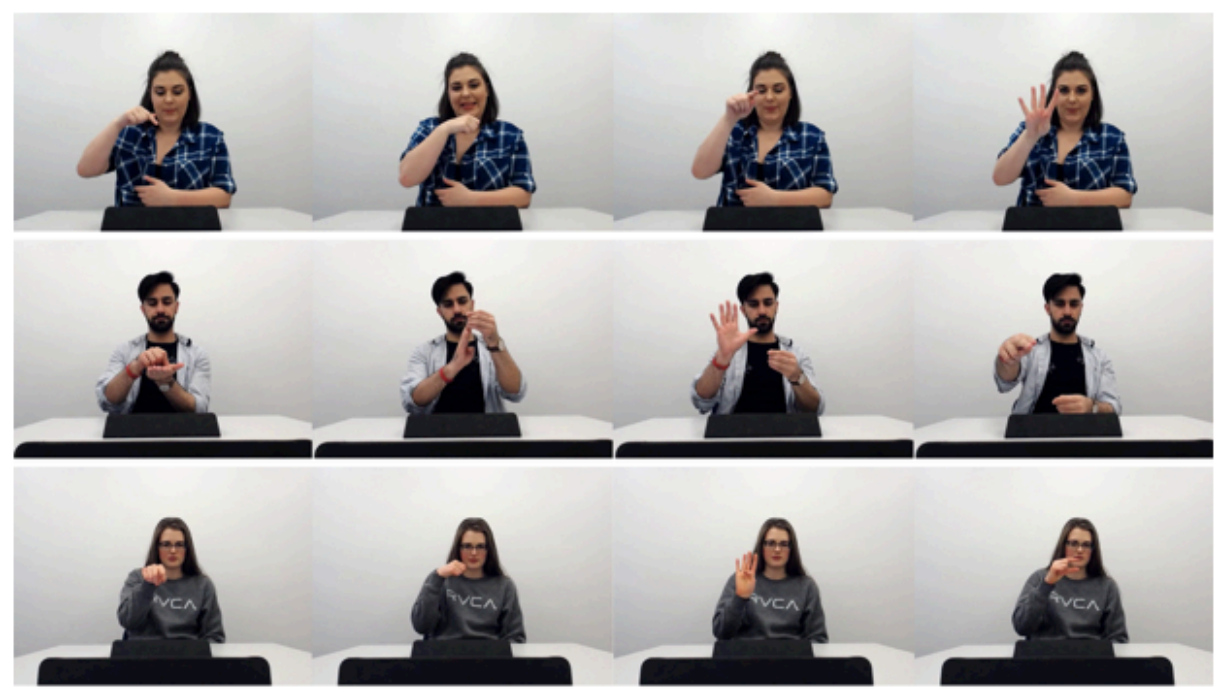

Figure 7: Clips from three participants illustrating example gestures (Top: texture condition, stimulus "four spotted toothbrushes" on proximal iPad, order Dem-N-Adj-Num; Middle: texture condition, stimulus "five striped pencils" on distal iPad, order N-Adj-Num-Dem; Bottom: size condition, stimulus "four small toothbrushes" on distal iPad, order Dem-N-Num-Adj).

\subsubsection{Coding}

Example gesture clips for Experiment 2 are shown in fig. 7. Gesture strings were coded as for Experiment 1. Agreement on gesture order was $90 \%$, on gesture homomorphism was 100\%. All instances of disagreement were resolved by discussion with a third coder.

\subsection{Results}

As in Experiment 1, gesture strings were overwhelmingly homomorphic, across both conditions $18 \%$ were non-homomorphic ( $1 \%$ were ambiguous e.g., due to repetitions). The most commonly used patterns, shown in fig. 8, were Dem-Num-N-A (approximately 400 gestures), Dem-Num-A-N, Dem-N-A-Num and Dem-N-Num-A (ranging from 150-185 each). This includes the English order (but not the reverse for example), suggesting some influence of the native language. Participants' self-reports indicate that few were consciously aware of using English order; very few participants reported that they produced gestures based on how they would have said it in English, most reported having no idea why they used a particular order, or putting the most important or salient information first. The most common orders produced using speech post-experiment were DemNum-N-A, Num-A-N-Dem, Dem-Num-A-N and Num-A-N (with Dem omitted). These overlap partially, but not entirely, with the gesture orders overall and there is a partial correspondence between the speech order participants used and their gesture order. For example, of the nine participants who produced an order resembling English in speech ((Dem)-Num-A-N), four used this in gesture as well, while the remaining 5 used a different order (e.g., Dem-Num-N-Num-A, Dem-N-A-Num, Dem-Num-N-A).

Returning to the question of interest here, fig. 8 suggests that more non-homomorphic orders were produced by participants in the low PMI size condition. Importantly, the most frequent of these involves reversing the order of $\mathrm{A}$ and Num, exactly as predicted. In the texture condition $89 \%$ of gestures were homomorphic ( $11 \%$ non); in the size condition $74 \%$ were homomorphic $(25 \%$ non). A logistic mixed-effect model with participant as a random effect revealed a significant overall preference for homomorphic order but no difference between conditions, either overall or for A and Num specifically (sum contrast coding, overall scope: intercept $\beta=3.08 \pm 0.53, p<0.001$, condition 

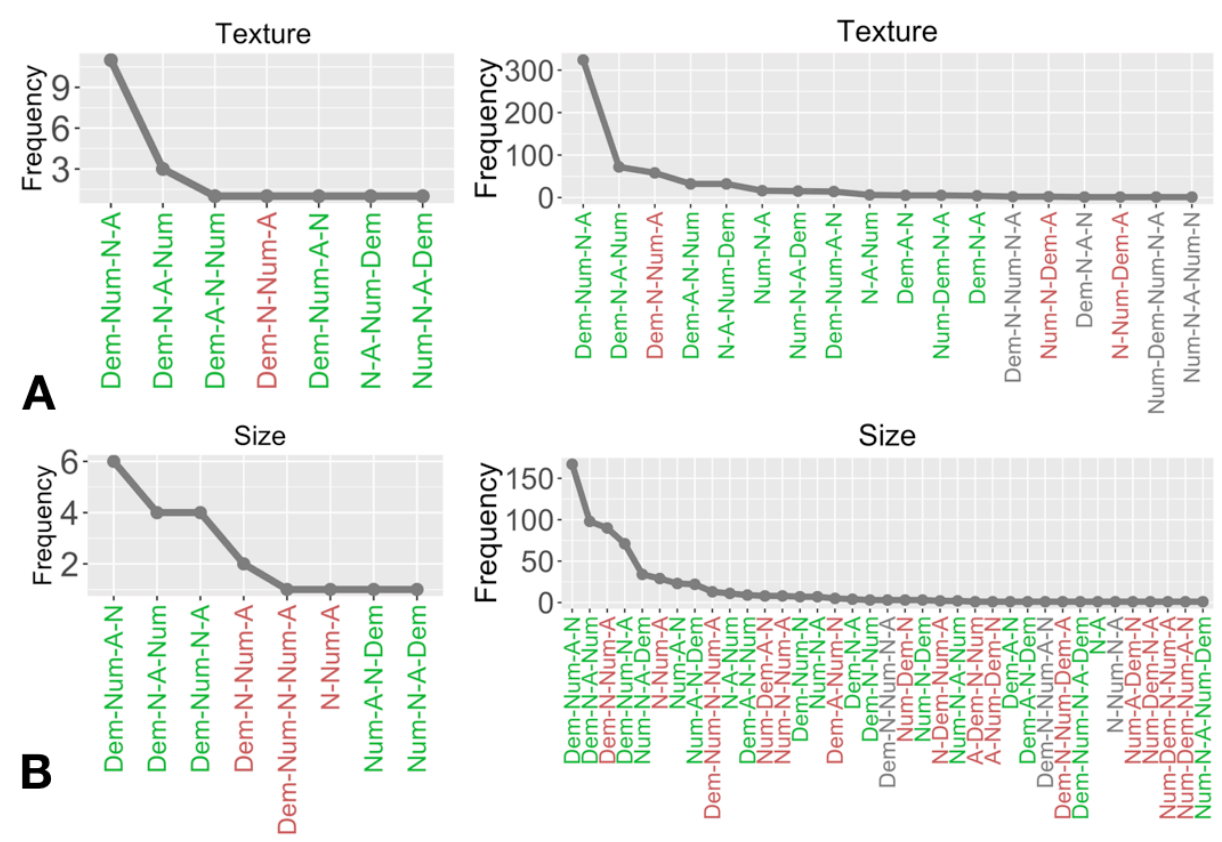

Figure 8: Number of participants (y-axis) using each majority pattern (x-axis) for texture condition (A) and size condition (B) The overwhelming majority of orders produced were homomorphic, with more non-homomorphic orders in the size condition.

$\beta=-0.63 \pm 0.50, p=0.20 ; \mathrm{A}$ and Num scope: intercept $\beta=3.48 \pm 0.64, p<0.001$, condition $\beta=-0.90 \pm 0.57, p=0.11$ ). Comparing these results to Experiment 1, there was no difference in use of homomorphic orders between the two texture conditions $(\beta=-1.6 \pm 1.12, p=0.15)$, but a significant difference between homomorphism in Experiment 1 (texture only) and the size condition in Experiment $2(\beta=-2.89 \pm 1.09, p<0.01)$. A comparison between the two texture conditions combined and the size condition in Experiment 2 also revealed a significant difference in homomorphism $(\beta=-0.96 \pm 0.44, p=0.03)$. In both cases, homomorphic orders were less likely to be used in the size condition. However, the pressure to use an homomorphic order remains strong across conditions.

\subsection{Discussion}

Noun phrase word order patterns documented in the world's languages follow a highly skewed distribution: a small number of patterns are very common while others are rare or as yet unattested. In previous research, linguists have claimed that this distribution-or more accurately, the fact that some patterns are unattested-is evidence for the influence of universal organizing principles, potentially innate and specialized for language (Cinque, 2005; Abels \& Neeleman, 2012; Steddy \& Samek-Lodovici, 2011; Dryer, 2018; Steedman, 2018). Here we have argued that universal organizing principles are indeed active in this domain, however they are likely cognition-general, and where they interface with the linguistic system they may be learnable. In particular, using an information-theoretic measure of strength of association computed on natural language corpora as a proxy for the real world, we showed that objects (expressed by Nouns) and their properties (expressed by Adjectives) are more closely related than objects and their numerosities (expressed by Numerals), which are in turn more closely related than objects and their location or relation to the speaker (expressed by Demonstratives). Specifically, average pointwise mutual information differed among these distinct types, with (Noun, Adjective) pairs consistently highest, and (Noun, Demonstrative) pairs lowest. This information is therefore available in the environment to form 
the basis of our conceptual knowledge of these elements. If the learned conceptual structure is such that properties conveyed by adjectives combine with objects first, and information conveyed by demonstratives last, then an independent universal organizing principle - homomorphism - will exert a pressure to map transparently onto word order.

In line with previous work using artificial (spoken) language experiments (Culbertson \& Adger, 2014; A. Martin et al., to appear; A. Martin et al., submitted), a bias for homomorphism in ordering the conceptual information expressed in the noun phrase was found in our silent gesture experiments. Participants' gesture sequences, improvised in a modality distinct from their previous language experience, were overwhelmingly homomorphic. To confirm the relationship between conceptual closeness and linear order using a more fine-grained distinction, our second gesture experiment manipulated types of properties (conveyed by size and texture adjectives). When the property to be conveyed was on average more similar in conceptual closeness to numerosity (size), this resulted qualitatively in more violations of homomorphism. This effect only reached significance when combined with the data from Experiment 1, confirming again the strength of the homomorphism bias.

While these experiments suggest that homomorphism directly links conceptual structure and linear order, in natural language the link may be mediated by semantic and/or syntactic representations. For example, conceptual representations may influence the development of subsequent linguistic representations-of how these elements are categorized, how they compose semantically, and how they form syntactic constituents. It may be that the bias for homomorphism targets these linguistic representations, rather than the conceptual structure. After all, syntactic categories like Noun, Adjective, Numeral, and Demonstrative are linguistic notions and rules determining linear order in a given language will target these.

We have argued here that conceptual closeness-in particular, differences in how objects relate to properties, numerosities, and location/relation to the speaker-is the ultimate driver of noun phrase word order patterns. However, recent work on word order and language processing suggests that mutual information derived from surface linguistic input (rather than observing the world) may also influence word order directly. Most recently, Hahn, Degen, Goodman, Jurafsky, and Futrell (2018) show that mutual information contributes to explaining adjective ordering preferences gathered from English speakers; when multiple adjectives are present in a phrase, the adjective with higher mutual information tends to be closer to the noun. Following Futrell and Levy (2017), this could be driven by memory-constrained incremental processing. Placing modifier-noun pairs with high mutual information far apart from one another increases processing effort. This is intriguing work, but only deals with adjective order. Nevertheless it would be interesting to explore the empirical predictions it would make for the order of other modifiers, and whether it might provide an alternative or complementary approach to noun phrase order grounded primarily in incremental processing considerations rather than conceptual structure.

To conclude, both the diversity and the similarities among patterns in this simple linguistic domain are critically important. The similarities allow us to relate features of language to general features of cognition, filtered through linguistic representations. We have argued that the pressure for transparent mappings (here between conceptual structure and linear order) leads languages to favor a particular set of noun phrase orders-with the adjective closest to, and the demonstrative farthest away from the noun. There are eight such orders, and they are all among the most robustly attested in the world's language. This general pressure for homomorphism reflects a type of simplicity, often taken to be a unifying principle of cognitive science (Chater \& Vitányi, 2003; Culbertson \& Kirby, 2016). The diversity of patterns actually found illuminates the probabilistic nature of the mechanisms linking cognition and linguistic structure, namely cultural evolution. More generally we hope we have demonstrated that long-standing puzzles about the origins of 
language structure can, and indeed should, be addressed through a combination of detailed survey of the world's languages, controlled investigations in the lab, and statistical analysis of large-scale cross-linguistic datasets reflecting actual language use. 


\section{References}

Abels, K. \& Neeleman, A. (2012). Linear asymmetries and the LCA. Syntax, 15(1), 25-74.

Adger, D. (2003). Core syntax. Oxford: Oxford University Press.

Alexiadou, A., Haegeman, L., \& Stavrou, M. (2007). Noun Phrase in the generative perspective. Berlin: Mouton de Gruyter.

Bouchard, D. (2002). Adjectives, number and interfaces: Why languages vary. Amsterdam: Elsevier.

Champollion, L. (2006). A game-theoretic account of adjective ordering restrictions. Ms., University of Pennsylvania, 1-16.

Chater, N. \& Vitányi, P. (2003). Simplicity: A unifying principle in cognitive science? Trends in Cognitive Sciences, 7(1), 19-22.

Cinque, G. (1993). On the evidence for partial N-movement in the Romance DP. University of Venice Working Papers in Linguistics, 3(2), 21-40.

Cinque, G. (2005). Deriving Greenberg's Universal 20 and its exceptions. Linguistic Inquiry, 36(3), $315-332$.

Culbertson, J. \& Adger, D. (2014). Language learners privilege structured meaning over surface frequency. Proceedings of the National Academy of Sciences, 111(16), 5842-5847.

Culbertson, J. \& Kirby, S. (2016). Simplicity and specificity in language: Domain general biases have domain specific effects. Frontiers in Psychology, 6(1964).

Culbertson, J., Smolensky, P., \& Legendre, G. (2012). Learning biases predict a word order universal. Cognition, 122, 306-329.

Culbertson, J., Smolensky, P., \& Wilson, C. (2013). Cognitive biases, linguistic universals, and constraint-based grammar learning. Topics in Cognitive Science, 5(3), 392-424.

Cysouw, M. (2010). Dealing with diversity: Towards an explanation of NP-internal word order frequencies. Linguistic Typology, $14(2), 253-287$.

Dryer, M. (2018). On the order of demonstrative, numeral, adjective and noun. Language, 94(4), $798-833$.

Elbourne, P. (2008). Demonstratives as individual concepts. Linguistics and Philosophy, 31(4), 409-466.

Futrell, R., Hickey, T., Lee, A., Lim, E., Luchkina, E., \& Gibson, E. (2015). Cross-linguistic gestures reflect typological universals: A subject-initial, verb-final bias in speakers of diverse languages. Cognition, 136, 215-221.

Futrell, R. \& Levy, R. (2017). Noisy-context surprisal as a human sentence processing cost model. In Proceedings of the 15th conference of the european chapter of the association for computational linguistics: Volume 1, long papers (pp. 688-698).

Gibson, E., Piantadosi, S. T., Brink, K., Bergen, L., Lim, E., \& Saxe, R. (2013). A noisy-channel account of crosslinguistic word-order variation. Psychological science, 0956797612463705.

Goldin-Meadow, S., So, W. C., Özyürek, A., \& Mylander, C. (2008). The natural order of events: How speakers of different languages represent events nonverbally. Proceedings of the National Academy of Sciences, 105(27), 9163-9168.

Hahn, M., Degen, J., Goodman, N. D., Jurafsky, D., \& Futrell, R. (2018). An information-theoretic explanation of adjective ordering preferences. In Proceedings of the 40 th annual conference of the cognitive science society.

Hall, M. L., Mayberry, R. I., \& Ferreira, V. S. (2013). Cognitive constraints on constituent order: Evidence from elicited pantomime. Cognition, 129(1), 1-17.

Hurford, J. R. (1987). Language and number: The emergence of a cognitive system. Oxford: Basil Blackwell. 
Jurafsky, D. \& Martin, J. H. (to appear). Speech and language processing: An introduction to natural language processing, computational linguistics, and speech recognition (3rd). Prentice Hall.

Kratzer, A. \& Heim, I. (1998). Semantics in generative grammar. Blackwell Oxford.

Martin, A., Abels, K., Adger, D., \& Culbertson, J. (submitted). Experiment evidence for the influence of structure and meaning on linear order in the noun phrase.

Martin, A., Abels, K., Ratitamkul, T., \& Culbertson, J. (to appear). Cross-linguistic evidence for cognitive universals in the noun phrase. Linguistics Vanguard.

Martin, J. E. (1969). Semantic determinants of preferred adjective order. Journal of Verbal Learning and Verbal Behavior, 8(6), 697-704.

Padden, C., Hwang, S.-O., Lepic, R., \& Seegers, S. (2015). Tools for language: Patterned iconicity in sign language nouns and verbs. Topics in Cognitive Science, 7(1), 81-94.

Partee, B. (1988). Many quantifiers. In Proceedings of escol (Vol. 5, pp. 383-402).

Partee, B. H. (1987). Noun phrase interpretation and type-shifting principles. In J. Groenendijk, D. de Jongh, \& M. Stokhof (Eds.), Studies in discourse representation theory and the theory of generalized quantifiers (pp. 115-143). Dordrecht: Foris.

Piantadosi, S. T. \& Gibson, E. (2014). Quantitative standards for absolute linguistic universals. Cognitive Science, 38, 736-756.

Rijkhoff, J. [J.]. (1990). Explaining word order in the Noun Phrase. Linguistics, 28(1), 5-42.

Rijkhoff, J. [Jan]. (2004). The noun phrase. Oxford: Oxford University Press.

Rubino, C. R. G. (2000). Ilocano dictionary and grammar: Ilocano-english, english-ilocano. University of Hawaii Press.

Saffran, J. R. (2003). Statistical language learning mechanisms and constraints. Current directions in psychological science, 12(4), 110-114.

Sagae, K., Davis, E., Lavie, A., MacWhinney, B., \& Wintner, S. (2007). High-accuracy annotation and parsing of childes transcripts. In Proceedings of the workshop on cognitive aspects of computational language acquisition (pp. 25-32). Association for Computational Linguistics.

Schouwstra, M. \& de Swart, H. (2014). The semantic origins of word order. Cognition, 131(3), $431-436$.

Schouwstra, M., Smith, K., \& Kirby, S. (2016). From natural order to convention in silent gesture. In S. Roberts, C. Cuskley, L. McCrohon, L. Barceló-Coblijn, O. Feher, \& T. Verhoef (Eds.), The evolution of language.

Scontras, G., Degen, J., \& Goodman, N. D. (2017). Subjectivity predicts adjective ordering preferences. Open Mind: Discoveries in Cognitive Science, 1(1), 53-66.

Scott, G.-J. (2002). Stacked adjectival modification and the structure of nominal phrases. In G. Cinque (Ed.), Functional structure in dp and ip. the cartography of syntactic structures (Vol. 1, pp. 91-120). Oxford: Oxford University Press.

Seiler, H. (1991). Determination: A functional dimension for interlanguage comparison. In H. Seiler (Ed.), Language universals, papers from the conference held at gummersbach/cologne, germany, october 3-8, 1976 (pp. 301-328). Tübingen: Narr.

Steddy, S. \& Samek-Lodovici, V. (2011). On the ungrammaticality of remnant movement in the derivation of Greenberg's Universal 20. Linguistic Inquiry, 42(3), 445-469.

Steedman, M. (2018). A formal universal of natural language grammar. Manuscript, University of Edinburgh.

Truswell, R. (2009). Attributive adjectives and nominal templates. Linguistic Inquiry, 40(3), 525533. 

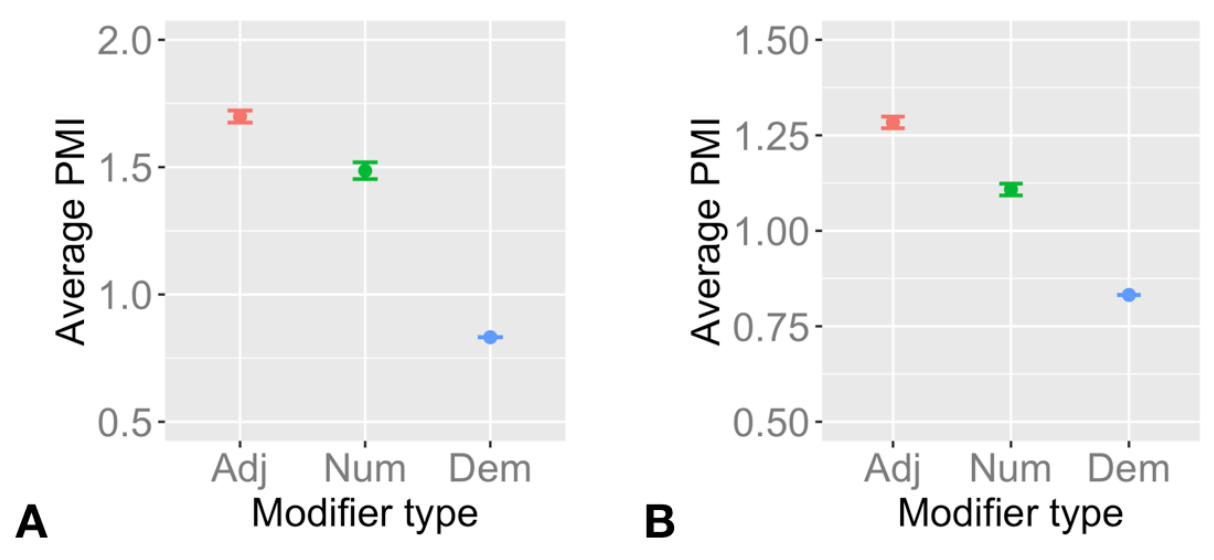

Figure A.9: A Average PMI values for 1000 samples of each modifier type, with set size fixed at 4 . This means demonstratives are always the same, but adjectives and numerals are randomly sampled from the unique set of each type. B Average PMI values for 1000 entropy matched samples of each modifier type, set size again fixed to 4 .

\section{Appendix A. Additional information about PMI calculations}

Adjectives, numerals and demonstratives differ from one another in terms of the size of the linguistic class; there are typically more adjectives than numerals, and a relatively small set of demonstratives. For example, English uses four (encoding distal/proximate and singular/plural distinctions), Latin used six (differentiating "near to me" and "near to you" in addition to "far away"), and other languages like Ilocano have a much larger set (making many distinctions in terms of visibility, continued existence, etc.; Rubino, 2000). But most languages tend to use a small number of demonstratives relative to adjectives and numerals. These categories themselves therefore reflect how the world is carved up by the human mind, in way that are specific to particular languages, and in ways that are likely not. Intuitively though, even if one were to vastly increase the number the of demonstratives - e.g., making ever finer distinctions in terms of physical or temporal distance - they will still be relative to the speaker. However, modifier types with fewer category members will typically have lower entropy, and in turn this could lower their PMI since the mutual information of a pair is bounded above by the entropy of the lower-entropy element. Note however, for some languages, the set of adjectives and numerals is similar in size, and yet still the PMI values differ (e.g., in our Indonesian corpus, there are 125 unique adjectives and 123 unique numerals, but adjective PMIs are still almost twice as high). Although our hypothesis posits a connection between linear order and PMI (not modifier set size or entropy), we checked whether entropy rather than PMI explains the differences between modifier types in our data. To do this, we randomly sampled small sets of adjectives and numerals from our English corpora, and calculated PMIs for each sample (1000 samples, set size $=4$, same as Dem). While entropies go down overall as expected, the PMI of Adj,N pairs is still highest, and the PMI for Dem,N the lowest. We also randomly sampled sets holding entropy near constant (a tight range around the entropy for demonstratives in our corpus), and our PMI differences still hold there as well. See A.9.

Average PMI values for color/texture vs. size adjectives were calculated by extracting the PMI values calculated as above for adjectives that matched these types. Adjectives were identified from lists of color, texture (including material) and size adjectives and then supplemented by hand coding. We also computed PMI values for these adjectives a second way: by re-computing PMI values such that the probability of a given modifier is based on the set of modifiers of that type (i.e., here color or size rather than adjective) modifying a head noun, and the probability of the noun is based on the set of nouns that have that type of modifier. This does not change the result, 
namely that PMI values for color/texture are higher than size.

\section{Appendix B. Additional information about corpora}

All adult-directed corpora are from the Universal Dependencies Treebank 2.0 Nivre et al., 2018. English child-directed speech data are the dependency-parsed English corpora available on CHILDES (Sagae, Davis, Lavie, MacWhinney, \& Wintner, 2007). We use all languages with available corpora totally more than 200k words (CHILDES is the largest), plus Basque and Indonesian (approximately $150 \mathrm{~K}$ each) in order to increase the diversity of the set. All treebank corpora used are listed in B.1.

Information about word order is not encoded directly in the dependencies, and the overwhelming majority of phrases containing one of these three modifier types in the corpora have only a single modifier (83\% in the English treebanks, compared to $14 \%$ with two modifiers, and $3 \%$ with three or more), further, almost all phrases (94\%) have only a single modifier type. Note that demonstratives are tagged inconsistently across treebank corpora. We extracted them using featural information first, and then manually checked them against grammars for each language to assemble the final set. Numeral dependencies are also tagged somewhat inconsistently across corpora (e.g., dates are sometimes tagged as cardinal numerals, some compound number words, e.g., "two hundred" are occasionally treated as two distinct words). We programmatically cleaned up the numeral dependencies where this was possible.

\section{References}

Ahrenberg, L. (2015). Converting an english-swedish parallel treebank to universal dependencies. In Third international conference on dependency linguistics (depling 2015), uppsala, sweden, august 24-26, 2015 (pp. 10-19). Association for Computational Linguistics.

Aranzabe, M. J., Atutxa, A., Bengoetxea, K., Diaz, A., de Ilarraza, I. G., Gojenola, K., \& Uria, L. (2014). Automatic conversion of the basque dependency treebank to universal dependencies. In Proceedings of the workshop on treebanks and linguistic theories (tlt 2014) (pp. 233-241).

Bamman, D. \& Crane, G. (2011). The ancient greek and latin dependency treebanks. In Language technology for cultural heritage (pp. 79-98). Springer.

Barbu Mititelu, V., Ion, R., Simionescu, R., Irimia, E., \& Perez, C. (2016). The romanian treebank annotated according to universal dependencies. In Proceedings of the tenth international conference on natural language processing (hrtal2016).

Bejček, E., Panevová, J., Popelka, J., Straňák, P., Ševčíková, M., Štěpánek, J., \& Žabokrtskỳ, Z. (2012). Prague dependency treebank 2.5-a revisited version of pdt 2.0. Proceedings of COLING 2012, 231-246.

Bies, A., Mott, J., Warner, C., \& Kulick, S. (2012). English web treebank, Linguistic Data Consortium, Philadelphia, PA.

Bobicev, V., Bumbu, T., Lazu, V., Maxim, V., \& Istrati, D. (2016). Folk poetry for computers: Moldovan codri's ballads parsing. In Proceedings of the 12th international conference "linguistic resources and tools for processing the romanian language (pp. 39-50).

Bosco, C., Lombardo, V., Lesmo, L., \& Daniela, V. (2000). Building a treebank for italian: A data-driven annotation schema. In Lrec 2000 (pp. 99-105). ELDA.

Bouma, G. \& Van Noord, G. (2017). Increasing return on annotation investment: The automatic construction of a universal dependency treebank for dutch. In Proceedings of the nodalida 2017 workshop on universal dependencies (udw 2017) (pp. 19-26). 
Table B.1: Corpora used

\begin{tabular}{|c|c|c|}
\hline Language & Family & Source \\
\hline French & IE, Germanic & $\begin{array}{l}\text { Turin University Parallel Treebank (Sanguinetti \& Bosco, } \\
\text { 2015), French UD Treebank (McDonald et al., 2013), Se- } \\
\text { quoia Treebank (Candito \& Seddah, 2012) }\end{array}$ \\
\hline Spanish & IE, Romance & $\begin{array}{l}\text { AnCora Treebank (Recasens \& Martí, 2010), Spanish UD } \\
\text { Treebank (McDonald et al., 2013) }\end{array}$ \\
\hline Catalan & IE, Romance & AnCora Treebank (Recasens \& Martí, 2010) \\
\hline Italian & IE, Romance & $\begin{array}{l}\text { Italian UD Treebank (Bosco, Lombardo, Lesmo, \& Daniela, } \\
\text { 2000), Turin University Parallel Treebank (Sanguinetti \& } \\
\text { Bosco, 2015), PoSTWITA-UD (Sanguinetti, Bosco, Alberto, } \\
\text { Mazzei, \& Fabio, 2018) }\end{array}$ \\
\hline Portuguese & IE, Romance & $\begin{array}{l}\text { UD Portuguese Treebank (Rademaker et al., 2017), Por- } \\
\text { tuguese UD Treebank (McDonald et al., 2013) }\end{array}$ \\
\hline Romanian & IE, Romance & $\begin{array}{l}\text { Romanian UD Treebank (Barbu Mititelu, Ion, Simionescu, } \\
\text { Irimia, \& Perez, 2016), Romanian Non-standard UD Tree- } \\
\text { bank (Bobicev, Bumbu, Lazu, Maxim, \& Istrati, 2016) }\end{array}$ \\
\hline Latin & IE, Romance & $\begin{array}{l}\text { UD Latin PROIEL Treebank (Haug \& Jøhndal, 2008), In- } \\
\text { dex Thomisticus Treebank (Cecchini, Passarotti, Marongiu, } \\
\text { \& Zeman, 2018), Perseus UD Latin Treebank (Bamman \& } \\
\text { Crane, 2011) }\end{array}$ \\
\hline Russian & IE, Slavic & $\begin{array}{l}\text { Russian Universal Dependencies Treebank (McDonald et al., } \\
\text { 2013), SynTagRus (Dyachenko et al., 2015), UD Russian } \\
\text { Taiga (Lyashevkaya et al., 2016) }\end{array}$ \\
\hline Czech & IE, Slavic & $\begin{array}{l}\text { Czech PDT UD Treebank (Bejček et al., 2012), Czech CAC } \\
\text { UD Treebank (Hladká et al., 2008), FicTree (Jelínek, 2017), } \\
\text { Parallel Universal Dependencies, Czech CLTT UD Treebank } \\
\text { (Vincent Kríž \& Urešová, 2015) }\end{array}$ \\
\hline Polish & IE, Slavic & $\begin{array}{l}\text { LFG Enhanced UD Treebank of Polish (Patejuk \& } \\
\text { Przepiórkowski, 2018), UD Polish Treebank (Wróblewska \& } \\
\text { Przepiórkowski, 2014) }\end{array}$ \\
\hline Hindi & IE, Indic & Hindi UD Treebank (Palmer et al., 2009) \\
\hline $\begin{array}{l}\text { Ancient } \\
\text { Greek }\end{array}$ & IE, Greek & $\begin{array}{l}\text { Perseus Universal Dependencies Greek Treebank (Bamman } \\
\& \text { Crane, 2011), UD Ancient Greek PROIEL (Haug \& } \\
\text { Jøhndal, 2008)= }\end{array}$ \\
\hline
\end{tabular}


Table B.2: Corpora used (con’t)

\begin{tabular}{|c|c|c|}
\hline Language & Family & Source \\
\hline English & IE, Germanic & $\begin{array}{l}\text { Turin University Parallel Treebank (Sanguinetti \& Bosco, } \\
\text { 2015), Georgetown University Multilayer corpus (Zeldes, } \\
\text { 2017), English Web Treebank (Bies, Mott, Warner, \& } \\
\text { Kulick, 2012), Parallel Universal Dependencies Treebank, } \\
\text { LinES Parallel Treebank (Ahrenberg, 2015) }\end{array}$ \\
\hline German & IE, Germanic & German UD Treebank (McDonald et al., 2013) \\
\hline Swedish & IE, Germanic & $\begin{array}{l}\text { LinES Parallel Treebank (Ahrenberg, 2015), Swedish- } \\
\text { Talbanken Treebank (Nivre \& Bandmann Megyesi, 2007) }\end{array}$ \\
\hline Dutch & IE, Germanic & $\begin{array}{l}\text { UD Dutch Alpino Treebank (Van der Beek, Bouma, Malouf, } \\
\& \text { Van Noord, 2002) UD Lassy Small Treebank (Bouma \& } \\
\text { Van Noord, 2017) }\end{array}$ \\
\hline Norwegian & IE, Germanic & $\begin{array}{l}\text { Norwegian UD Treebank, Bokml, Nynorsk (Velldal, Øvrelid, } \\
\& \text { Hohle, 2017), LIA Norwegian UD Treebank (Øvrelid \& } \\
\text { Hohle, 2016) }\end{array}$ \\
\hline Estonian & Uralic, Finnic & UD Estonian Treebank (Muischnek et al., 2014) \\
\hline Finnish & Uralic, Finnic & $\begin{array}{l}\text { UD FinnTreeBank } 1 \text { (Hakulinen et al., 2004), UD Turku } \\
\text { Dependency Treebank (Haverinen et al., 2014) }\end{array}$ \\
\hline Chinese & Sino-Tibetan & Traditional Chinese UD Treebank (McDonald et al., 2013) \\
\hline Korean & Korean & $\begin{array}{l}\text { Korean UD Treebank (McDonald et al., 2013), KAIST Ko- } \\
\text { rean UD Treebank (Chun, Han, Hwang, \& Choi, 2018) }\end{array}$ \\
\hline Arabic & $\begin{array}{l}\text { Afro-Asiatic, } \\
\text { Semitic }\end{array}$ & $\begin{array}{l}\text { Arabic-PADT UD Treebank (Smrž et al., 2008), NYUAD } \\
\text { Arabic UD treebank (Maamouri, Bies, Buckwalter, Jin, \& } \\
\text { Mekki, 2005) }\end{array}$ \\
\hline Japanese & Japanese & Japanese UD Treebank (McDonald et al., 2013) \\
\hline Indonesian & $\begin{array}{l}\text { Austronesian, } \\
\text { Malayo- } \\
\text { Sumbawan }\end{array}$ & Indonesian UD Treebank (McDonald et al., 2013) \\
\hline Basque & Basque & Basque UD Treebank (Aranzabe et al., 2014) \\
\hline
\end{tabular}


Candito, M. \& Seddah, D. (2012). Le corpus sequoia: Annotation syntaxique et exploitation pour l'adaptation d'analyseur par pont lexical. In Taln 2012-19e conférence sur le traitement automatique des langues naturelles.

Cecchini, F. M., Passarotti, M., Marongiu, P., \& Zeman, D. (2018). Challenges in Converting the Index Thomisticus treebank into Universal Dependencies. Proceedings of the Universal Dependencies Workshop 2018 (UDW 2018).

Chun, J., Han, N.-R., Hwang, J. D., \& Choi, J. D. (2018). Building universal dependency treebanks in korean. In Proceedings of the 11th international conference on language resources and evaluation, lrec'18, miyazaki, japan, 2018.

Dyachenko, P., Iomdin, L., Lazursky, A., Mityushin, L., Podlesskaya, O., Sizov, S., .. Tsinman, L. (2015). The current state of the deeply annotated corpus of the texts of the russian language (sintagrus). Proceedings of the Russian Language Institute. (6), 272-300.

Hakulinen, A., Vilkuna, M., Korhonen, R., Koivisto, V., Tarja, R. H., \& Alho, I. (2004). Iso suomen kielioppi. Helsinki: Suomalaisen Kirjallisuuden Seura.

Haug, D. T. \& Jøhndal, M. (2008). Creating a parallel treebank of the old indo-european bible translations. In Proceedings of the second workshop on language technology for cultural heritage data (latech 2008) (pp. 27-34).

Haverinen, K., Nyblom, J., Viljanen, T., Laippala, V., Kohonen, S., Missilä, A., .. Ginter, F. (2014). Building the essential resources for finnish: The turku dependency treebank. Language Resources and Evaluation, 48(3), 493-531.

Hladká, B., Hajič, J., Hana, J., Hlaváčová, J., Mírovskỳ, J., \& Raab, J. (2008). The czech academic corpus 2.0 guide. The Prague Bulletin of Mathematical Linguistics, 89, 41-96.

Jelínek, T. (2017). Fictree: A manually annotated treebank of czech fiction. In J. Hlaváčová (Ed.), Itat 2017 proceedings (pp. 181-185).

Lyashevkaya, O., Droganova, K., Zeman, D., Alexeeva, M., Gavrilova, T., Mustafina, N., \& Shakurova, E. (2016). Universal dependencies for russian: A new syntactic dependencies tagset. Linguistics, WP BRP 44/LNG/2016.

Maamouri, M., Bies, A., Buckwalter, T., Jin, H., \& Mekki, W. (2005). Arabic treebank: Part 3 (full corpus) v 2.0 (mpg + syntactic analysis). Linguistic Data Consortium, Philadelphia, lDC Catalogue number: LDC2005T20, ISBN.

McDonald, R., Nivre, J., Quirmbach-Brundage, Y., Goldberg, Y., Das, D., Ganchev, K., .. Täckström, O., et al. (2013). Universal dependency annotation for multilingual parsing. In Proceedings of the 51st annual meeting of the association for computational linguistics (volume 2: Short papers) (Vol. 2, pp. 92-97).

Muischnek, K., Müürisep, K., Puolakainen, T., Aedmaa, E., Kirt, R., \& Särg, D. (2014). Estonian dependency treebank and its annotation scheme. In Proceedings of 13th workshop on treebanks and linguistic theories (tlt13) (pp. 285-291).

Nivre, J., Abrams, M., Agić, Ž., Ahrenberg, L., Antonsen, L., Aranzabe, M. J., ... Zhu, H. (2018). Universal dependencies 2.2. LINDAT/CLARIN digital library at the Institute of Formal and Applied Linguistics (ÚFAL), Faculty of Mathematics and Physics, Charles University.

Nivre, J. \& Bandmann Megyesi, B. (2007). Bootstrapping a swedish treebank using cross-corpus harmonization and annotation projection. In Proceedings of the 6th international workshop on treebanks and linguistic theories (pp. 97-102).

Øvrelid, L. \& Hohle, P. (2016). Universal dependencies for norwegian. In Proceedings of the ninth international conference on language resources and evaluation (lrec'16).

Palmer, M., Bhatt, R., Narasimhan, B., Rambow, O., Sharma, D. M., \& Xia, F. (2009). Hindi syntax: Annotating dependency, lexical predicate-argument structure, and phrase structure. In The 7th international conference on natural language processing (pp. 14-17). 
Patejuk, A. \& Przepiórkowski, A. (2018). From Lexical Functional Grammar to enhanced Universal Dependencies: Linguistically informed treebanks of Polish. Warsaw: Institute of Computer Science, Polish Academy of Sciences.

Rademaker, A., Chalub, F., Real, L., Freitas, C., Bick, E., \& de Paiva, V. (2017). Universal dependencies for portuguese. In Proceedings of the fourth international conference on dependency linguistics (depling) (pp. 197-206). Pisa, Italy.

Recasens, M. \& Martí, M. A. (2010). Ancora-co: Coreferentially annotated corpora for spanish and catalan. Language resources and evaluation, 44(4), 315-345.

Sagae, K., Davis, E., Lavie, A., MacWhinney, B., \& Wintner, S. (2007). High-accuracy annotation and parsing of childes transcripts. In Proceedings of the workshop on cognitive aspects of computational language acquisition (pp. 25-32). Association for Computational Linguistics.

Sanguinetti, M. \& Bosco, C. (2015). Parttut: The turin university parallel treebank. In Harmonization and development of resources and tools for italian natural language processing within the parli project (pp. 51-69). Springer.

Sanguinetti, M., Bosco, C., Alberto, L., Mazzei, A., \& Fabio, T. (2018). Postwita-ud: An italian twitter treebank in universal dependencies. In Language resources and evaluation conference 2018 (pp. 1-8). ELRA.

Smrž, O., Bielický, V., Kouřilová, I., Kráčmar, J., Hajič, J., \& Zemánek, P. (2008). Prague arabic dependency treebank: A word on the million words. Proceedings of the Workshop on Arabic and Local Languages (LREC 2008), 16-23.

Van der Beek, L., Bouma, G., Malouf, R., \& Van Noord, G. (2002). The alpino dependency treebank. Language and Computers, 45, 8-22.

Velldal, E., Øvrelid, L., \& Hohle, P. (2017). Joint ud parsing of norwegian bokmål and nynorsk. In Proceedings of the 21st nordic conference on computational linguistics, nodalida, 22-24 may 2017, gothenburg, sweden (131, pp. 1-10). Linköping University Electronic Press.

Vincent Kríž, B. H. \& Urešová, Z. (2015). Czech legal text treebank. LINDAT/CLARIN digital library at Institute of Formal and Applied Linguistics, Charles University in Prague.

Wróblewska, A. \& Przepiórkowski, A. (2014). Projection-based annotation of a Polish dependency treebank. In Proceedings of the ninth international conference on language resources and evaluation, lrec (pp. 2306-2312).

Zeldes, A. (2017). The gum corpus: Creating multilayer resources in the classroom. Language Resources and Evaluation, 51(3), 581-612. 